APPENDIX 2. Addresses of Participants.

Dr. Helmut A. Abt

Kitt Peak National Observatory Box 26732

Tucson, AZ 85726-6732

Ms. Vagiswari Alladi

Indian Institute of Astrophys.

Koramangala Bangalore 560034

INDIA

William E. Anderson

Case Western Reserve Univ.

Sears Library

10900 Euclid Ave.

Cleveland, $\mathrm{OH} 44106$

Jim Ashling

INSPEC/IEEE Service Center

445 Hoes Lane

P. O. Box 1331

Piscataway, NJ 08855-1331

Ms. Martha L. Austin

Physics-Astronomy Lib. FM-15

Univ. of Washington

Seattle, WA 98195

Dr. Maya Avasia

T.I.F.R. Housing Colony

"Panini-006"

Homi Bhabha Road

Colaba, Bombay 400005 INDIA

Michael Barylak

ESA IUE Observatory

P.B. 54065

28080 Madrid SPAIN

Judith Bausch

Yerkes Observatory

P.O. Box 258

Williams Bay, WI 53191

Chris Benn

Royal Greenwich Observatory

Herstmonceux Castle

Hailsham E. Sussex BN27 1RP

GREAT BRITAIN
Ms. Zuleika Berto

Instituto de Fisica

Campus do Vale

90049 Porto Alegre RS

BRAZIL

Olga B. Boemeke

Princeton University

Astrophys. Sciences Library

Peyton Hall

Princeton, NJ 08540

Ms. Ellen N. Bouton

NRAO Library

Edgemont Road

Charlottesville, VA 22803-2475

Dr. Peter B. Boyce

American Astronomical Society

Suite 300

2000 Florida Avenue N.W.

Washington, DC 20009

Dottie Brumbaugh

Lowell Observatory

Mars Hill Road 1400 W.

Flagstaff, AZ 86001

Ms. Elizabeth Bryson

Canada-France-Hawaii Telescope

PO Box 1597

Kamuela, HI 96743

Ron Buchan

NASA STI Facility

P.O. Box 8757

BWI Airport

Baltimore, MD 21240

Dr. W. Buscombe

Dearborn Obs.

Northwestern Univ.

Evanston, IL 60201

Merri Sue Carter

TSEO

U.S. Naval Observatory

Washington, DC 20392-5100
Francesco A. Catalano

Univ. of Catania-Inst. di Astron.

Citta Universitaria

Viale A. Doria

I-95125 Catania ITALY

Michael J. Collins

INSPEC

Station House Nightingale Road

Hitchin Hertfordshire

ENGLAND SG5 1RJ

Mrs. Brenda Corbin

Library

U.S. Naval Observatory

Washington, DC 20392-5100

Mo. Karen S. Croneis

Univ. of Texae at Austin

Physics-Math Astronomy Lib.

RLM 4.200

Austin, TX 78712-7330

Ms. Marlene Cummins

Astronomy Library

Univ. of Toronto-Rm. 1306

60 St. George St.

Toronto Ont. M5S 1A7 CANADA

M. Henri Paul Dale

Obs. Royal De Belgique

3 Avenue Circulaire

B-1180 Brussels BELGIUM

Ms. Jenny Davies

CSIRO Div. of Radiophysics

PO Box 76

Epping NSW 2121

AUSTRALIA

Dr. Suzanne Debarbat

Observatoire de Paris

61 avenue de l'Observatoire

75014 Paris FRANCE

Ms. Adelaide Del Frate

NASA Headquarters

Code NTT-1

Washington, DC 20546 
Jim DeYoung

Nautical Almanac Office

U.S. Naval Observatory

Washington, DC 20392-5100

Dr. Steven Dick

U.S. Naval Observatory

Washington, DC 20392-5100

M. Pauline DiGioia

Astronomy Libr. Yale Univ.

PO Box 6666

260 Whitney Ave.

New Haven, CT 06511

Dr. Olga B. Dluzhnevskaya

Astronomical Council

USSR Academy of Sciences

Pyatnitskaya UL 48

109017 Moscow U.S.S.R.

Dainis Dravins

Lund Observatory

Box 43

S-22100 Lund SWEDEN

Dr. Pascal Dubois

Centre des Donnees Stellaires

11 rue de l'Universite

67000 Strasbourg FRANCE

Ms. Janet Dudley

Royal Signals \& Radar Estab.

St. Andrews Road

Malvern Worcs. WR14 3PS

ENGLAND

Ms. Anne Fishburn

Max-Planck-Inst. fur Astrophys.

Karl-Schwaraschild-Str. 1

D-8046 Garching bei Munchen

F.R. GERMANY

Barbara A. Ford-Foster

Univ. of Calif-Irvine

P.O. Box 19557

Irvine, CA 92713
Ms. Ruth Freitag

Library of Congress

Science \& Technology Div.

Washington, DC 20540

Dr. Mikhail S. Frolov

Astronomical Council

USSR Academy of Sciences

Pyatnitskaya UL $\mathbf{4 8}$

108017 Moscow U.S.S.R.

Mary Fugle

Springer-Verlag New York

175 Fifth Avenue

New York, NY 10010

Ms. Reiko Fujita

Tokyo Astronomical

Observatory Library

Mitaka-shi Tokyo 181

JAPAN

Ms. Joan Gantz

Mt. Wilson \& Las Campanas Obs. Huang Bi-kun

Library

813 Santa Barbara Street

Pasadena, CA 91101

Mike Genuardi

NASA STI Facility

P.O. Box 8757

BWI Airport

Baltimore, MD 21240

\section{Barbara Gertner}

Inst. of Astr. \& Radio Astr. Copernicus Univ. in Torun

Chopina 12/18

PL 87-100 Torun POLAND

Ms. Maria Eugenia Gomez ESO Library-La Silla

Casilla 19001

Santiago 19 CHILE

Mrs. Selma S. de Guerra

Observ. Astron. de Cordoba

Laprida Street 854

5000 Cordoba ARGENTINA

MALAYSIA
Jean-Louis Halbwachs

Centre des Donnees Stellaires

11 rue de l'Universite

67000 Strasbourg FRANCE

Jan F. Hattink

Kluwer Academic Publishers

Spuiboulevard $\mathbf{5 0}$

P.O. Box 17

3900 Dordrecht

NETHERLANDS

Bambang Hidayat

Bosscha Observatory

Lembang 40391

West Java INDONESIA

Ms. Betty Holmstrom

Steward Observatory

Parker Room Library

Univ. of Arizona

Tucson, AZ 84721

Library

Purple Mountain Observatory

Nanking CHINA

Ms. Carol Hutchins

Swain Hall Libr. SW 208

Indiana Univ.

Bloomington, IN 47405

Dr. Mohammad Ilyas

School of Physics

Univ. of Science of Malaysia

11800 USM Penang

Ian Jordan

Astrometry Dept.

U.S. Naval Observatory

Washington, DC 20392-5100

Dr. Jun Jugaku

Tokai University

1117 Kitakaname

Hiratsuka-shi

Kanagawa 259-12 JAPAN 
Mrs. Kinga Kaminska

Astronomical Observatory of the Warsaw University

Al. Ujazdowskie 4

00-478 Warsaw POLAND

\section{Young Woon Kang}

Astronomical Data Center

Natl. Space Science Data Ctr.

NASA/GSFC - Code 633

Greenbelt, MD 20771

Dr. Frank J. Kerr

Astronomy Program

Univ. of Maryland

College Park, MD 20742

\section{Gert Kiers}

Kluwer Academic Publishers

Spuiboulevard 50

P.O. Box 17

3300AA Dordrecht

NETHERLANDS

Ms. Sandra E. Kitt

Richard S. Perkin Libr.

Amer. Mus.-Hayden Planetarium

81st St. \& Central Park West

New York, NY 10024

Helen Z. Knudsen

California Institute of Tech.

Astrophysics Library 105-24

Pasadena, CA 91125

Julie Kreunen

Physics-Optics-Astron. Libr.

B\&L 374

Univ. of Rochester

Rochester, NY 14627

Marek Krosniak

Astronomical Observatory of

the Jagiellonian University

Ul. Orla 171

30-244 Krakow POLAND
Janardan Kulkarni

Laura Kersey Library

Univ. of Louisville

Louisville, KY 40292

Ms. Suzanne Laloe

Institut d'Astrophysique

98 bis Boulevard Arago

75014 Paris FRANCE

Mrs. Ethleen Lastovica

South African

Astronomical Observatory

PO Box 9 Observatory

7935 SOUTH AFRICA

Eric S. LeBlane

Hertzberg Institute of Astrophys.

Dominion Astrophysical Obs.

5071 West Saanich Road RR 5

Victoria V8X 4M6 CANADA

De-He Li

Head - Information Dept.

Shaanxi Astronomical Observat.

PO Box 18 Lintong

Xian CHINA

Jinming Liu

Progress in Astronomy

80 Nandan Road

Shanghai

CHINA

J. Sainz Ballesteros Lloveras

Complejo Astronomico "EL

Leonciton

Casilla de Correo 467

5400 San Juan ARGENTINA

Ms. April M. Love

Univ. of Calif. Irvine

Physical Sciences Library

PO Box 19557

Irvine CA 92713

Donald A. Lubowich

American Institute of Physics 500 Sunnyside Boulevard

Woodbury, NY 11797
Wolfgang Lück

FIZ Karlsruhe

7514 Eggenstein

F. R. GERMANY

Angus R. Macdonald

Royal Observatory Edinburgh

Blackford Hill

Edinburgh EH9 $3 \mathrm{HJ}$ SCOTLAND

Robert H. Marks

Director of Publishing

American Institute of Physics

335 East 45th St

New York, NY 10017

Patricia Marshall

AIAA

Technical Information Service

555 West 57th Street

New York, NY 10019

Dr. Janet Mattei

AAVSO

25 Birch Street

Cambridge, MA 02138

Ms. Dorothy McGarry

Univ. of Calif. Los Angeles

8033 Sunset Blvd. \#4040

Los Angeles, CA 90046

Dr. Jaylee M. Mead

NASA Goddard Space Flt. Ctr.

Code 630

Greenbelt, MD 20771

Ms. Pat Molholt

Rensselaer Polytech. Institute

Folsom Library

Troy, NY 12180-3590

Tony Mollez

Public Affairs Office

U.S. Naval Observatory

Washington, DC 20392-5100 
Ms. A. M. Motais de Narbonne

Observatoire de Paris

61 Ave. de l'Observatoire

75014 Paris, FRANCE

Shiro Nishimura

National Astronomical Obs.

Mitake Tokyo 181

JAPAN

Francois Ochsenbein

European Southern Obn.

Karl-Schwarsschild Str. 2

D 8046 Garching bei Munchen

F. R. GERMANY

Steve Panossian

Nautical Almanac Office

U.S. Naval Observatory

Washington, DC 20392-5100

Bruce Pels

Univ. of Calif. Los Angeles

15931 Kalisher St.

Granada Hills, CA 91344

Ms. Alice Primack

Univ. of Floridn Libraries

Gainesville, FL 32611

Prof. Edoardo Proverbio

Osservatorio Astronomico

Via Ospedale 72

09100 Cagliari ITALY

\section{A. Ratnakar}

Raman Research Inst.

Sadashivanagar Post

Bangalore 560080 IN DIA

Perry W. Remaklus

Willmonn-Bell, Inc.

P.O. Box 35025

Richmond, VA 23235

Patricia B. Remaklus

Willmann-Bell, Inc.

P.O. Box 35025

Richmond, VA 23235
Ms. Joyce M. Rey-Watson

Smithsonian Astrophys. Obs.

60 Garden Street

Cambridge, MA 02138

Jane Riddle

Library - Code 252

NASA Goddard Space Flt. Ctr.

Greenbelt, MD 20771

Leif Robinson

Sky \& Telescope

49 Bay State Road

Cambridge, MA 02238-1290

Dr. Elisabeth Roemer

Lunar \& Planetary Laboratory

Univ. of Arizona

Tucson, AZ 85721

Mr. G. Russo

ESO ST-ECF

Karl-Schwarsschild Str. 2

D-8046 Garching Bei Munchen

F.R. GERMANY

Ms. Edith Sachtschal

ESO Library

Karl Schwarzschild Str. 2

D-8046 Garching bei Munchen

F.R. GERMANY

Dr. Luts D. Schmadel

Astronomisches Rechen-Inst.

Monchofstr. 12-14

D-6900 Heidelberg 1

F.R. GERMANY

Marion Schmit

IPAC 100-22

Calif. Institute of Technology

Pasadena, CA 91125

John T. Scott

American Institute of Physics

500 Sunnyside Blvd.

Woodbury, NY 11797
Andrei Radu Serban

Hamem-Gimmel Str. 27

Jerusalem 94422

ISRAEL

Mr. Gregory Shelton

Library

U.S. Naval Observatory

Wushington, DC 20392-5100

Mrs. Robyn Shobbrook

Anglo-Australian Observatory

P.O. Box 296

Epping NSW 2121

AUSTRALIA

David H. Stern

Physics/Astr. Library

204 Loomis Laboratory

1101 West Green Street

Urbana, IL 61801

Ms. Sarah Stevens-Rayburn

Space Telescope Science Inst.

3700 San Martin Drive

Baltimore, MD 21218

Ms. Kathryn Strand

High Altitude Obs./NCAR

PO Box 3000

Boulder, CO 80307

Mrs. Eva Stroot

Geneva Observatory Library

51 Ch. des Maillettes

1290 Sauverny SWITZERLAND

Ms. Cathy Van Atta

Kitt Peak Natl. Observatory

PO Box 26732

Tucson, AZ 85726

Mrs. M. Vargha

Konkoly Observatory

Box 67

1525 Budapest XII

HUNGARY 
Marie-Jose Vin

Observatoire de Haute Provence

FO4870 St. Michel l'Observatoire

FRANCE

Ms. Rosalind Walcott

SUNY at Stony Brook

Earth \& Space Sci. Library

Stony Brook, NY 11794-2199

\section{Ya-Hong Wang}

Shaanxi Astronomical Observ.

PO Box 18 Lintong

Xian CHINA

Dr. Wayne H. Warren

NASA-Goddard Space Flt. Ctr.

Code 633

Greenbelt, MD 20771

\section{Susan West}

Physics Library

Catholic University

Washington, DC 20064

Dr. Gart Westerhout

Scientific Director

US Naval Observatory

Washington, DC 20392-5100

Jack W. Weigel

Univ. of Michigan

205 Graduate Library

Ann Arbor MI 48109-1205

Dr. George A. Wilkins

Royal Greenwich Observatory

Herstmonceux Castle

Hailsham E. Sussex BN27 1RP

GREAT BRITAIN

Dr. Marek Wolf

Dept. of Aston. \& Astrophys.

Charles Univ. Prague

Svedaka 8

15000 Praha 5

CZECHOSLOVAKIA
Mr. Charles Worley

Astrometry Dept.

US Naval Observatory

Washington, DC 20392-5100

Allen Wynne

Math-Physics Library

Campus Box 184

Univ. of Colorado

Boulder, CO 80309

Zhou Yunfen

Academia Sinica

Yunnan Observatory

PO Box 110

Kunming CHINA

Dr. Josef Zavrel

Centre of Scientific Inf.

Astronomical Institute

25165 Ondrejov

CZECHOSLOVAKIA

Ms. Dorothea M. Zitta

Astronomy Program

Space Sciences Bldg.

Univ. of Maryland

College Park, MD 20742 